\title{
Classifying shoulder implants in X-ray images using Big data techniques
}

\author{
M. Sivachandran ${ }^{1}$, Dr.T.Krishnakumar ${ }^{2}$ \\ \{sivachandranm08@gmail.com ${ }^{1}$, drkk@bharathuniv.ac.in ${ }^{2}$ \} \\ Research Scholar, Department of CSE, Bharath Institute of Higher Education and Research, Chennai, \\ India. ${ }^{1}$, \\ Professor, Department of CSE, Bharath Institute of Higher Education and Research, Chennai, India. ${ }^{2}$
}

\begin{abstract}
This research work focuses on optimal solution for the image detection and segmentation. The prosthesis may - a few or numerous years after it was embedded come needing fix or substitution. In a portion of these cases, the maker and the model of the prosthesis might be obscure to the patients and their essential consideration specialists, for instance when the medical procedure was led in another nation where the patient has presently no admittance to the records. The highest accuracy value is $81.68 \%$ which is while applying the $=7 \mathrm{k}=6$ and the lowest accuracy value is $65.6 \%$ when apply the $\mathrm{k}=7$. The highest precision value is $81.68 \%$ while applying the $\mathrm{k}=1$ and very lowest precision value is $73.69 \%$ lies on $\mathrm{k}=2$ and $\mathrm{k}=9$. The highest recall value is $83.69 \%$ which is produced by while applying the parameter $\mathrm{k}=6$, the lowest recall value is $65.76 \%$ while applying the parameter $\mathrm{k}=9$. The $\mathrm{K}=10$ model takes more time to build the model is while applying the $\mathrm{k}=10$ and very low time consumption model is $\mathrm{k}=8$. Another conceivable instance of not knowing the specific producer and model could be expected uncertainty in clinical records or clinical images.
\end{abstract}

Keywords: KNN, Instance based classifier, Lazy classifier, TSA,UW.

\section{Introduction}

Total Shoulder Arthroplasty (TSA) is a typical intrusive system for treating harmed shoulder joints, where the shoulder ball is supplanted with a prosthesis.[1] The method is gone before and followed by a progression of X-beam pictures to evaluate arrangement and fit.[2] Common explanations behind going through TSA medical procedure are basic shoulder wounds or serious arthritis.[3,17] The technique mitigates torment and reestablishes movement to the patients shoulder.[4,16] There are a few distinct makers delivering prostheses, and every one of them offers a few unique models to all the more likely fit any sort of circumstance and patient.[5,15]The prosthesis may - a few or numerous years after it was embedded - come needing fix or replacement.[6,14] In a portion of these cases, the producer and the model of the prosthesis might be obscure to the patients and their essential consideration specialists, for instance when the medical procedure was directed in another nation where the patient has right now no admittance to the records.[7,13] Another conceivable instance of not knowing the specific maker and model could be expected uncertainty in clinical records or clinical images. $[8,12]$ right now, the errand of recognizing a prosthesis model in such cases is based on thorough assessments and visual correlations of X- 
beam pictures taken from the embed by clinical experts.[9,10,11] This can be a dreary assignment and requires time and exertion for each new patient.

In this paper presents in section 2 presents the materials and methods adopted and section 3 presents the details of the experiments and discussions. Finally section 4 concludes the paper by sharing our inferences and future plans.

\section{Materials And Methods}

The dataset gathered from UCI vault. Pictures were gathered by Maya Stark at BIDAL Lab at SFSU for her MS postulation project. They are from The UW Shoulder Site ([Web Link]), producer sites, and Feeley Lab at UCSF. The first assortment included $605 \mathrm{X}$-beam pictures. Eight pictures that seemed to have been taken from similar patients were eliminated, bringing about the last 597 pictures. The last set contains pictures from the accompanying makers: 83 from Cofield, 294 from Depuy, 71 from Tornier, and 149 from Zimmer, bringing about a 4-class arrangement issue. Class marks are given as the maker name in document names. Pictures are with 8-bit grayscale and different measurements in jpeg design. Weka 3.9.8.tool has implemented for the analysis and the below methods have implemented in this research work.

- Instance based classifier(KNN)

\section{Results And Discussions}

In this section focuses on the results and discussions of the instance based classifier implemented in this dataset.

Table 1: Instance based classifier Vs Accuracy

\begin{tabular}{|l|l|l|}
\hline S.No & K & Accuracy \\
\hline 1 & 1 & 79.49 \\
\hline 2 & 2 & 79.44 \\
\hline 3 & 3 & 74.81 \\
\hline 4 & 4 & 77.43 \\
\hline 5 & 5 & 73.69 \\
\hline 6 & 6 & 81.68 \\
\hline 7 & 7 & 65.6 \\
\hline 8 & 8 & 76.19 \\
\hline 9 & 9 & 77.43 \\
\hline 10 & 10 & 73.69 \\
\hline
\end{tabular}

The above table clearly shows that the instance based classifier is producing the accuracy levels are different while doing the parameter tuning like $\mathrm{K}$ parameter. In this dataset we implemented the lazy classifier while $\mathrm{K}=1$ then the system gets an accuracy value is $79.49 \%$, while $\mathrm{K}=2$ then the system gets an accuracy value is $79.44 \%$, while $\mathrm{K}=3$ then the system gets 
an accuracy value is $74.81 \%$, while $\mathrm{K}=4$ then the system gets an accuracy value is $77.43 \%$, while $\mathrm{K}=5$ then the system gets an accuracy value is $73.69 \%$, while $\mathrm{K}=6$ then the system gets an accuracy value is $81.68 \%$, while $\mathrm{K}=7$ then the system gets an accuracy value is $65.6 \%$, while $\mathrm{K}=8$ then the system gets an accuracy value is $76.19 \%$, while $\mathrm{K}=9$ then the system gets an accuracy value is $77.43 \%$, while $\mathrm{K}=10$ then the system gets an accuracy value is $73.69 \%$.

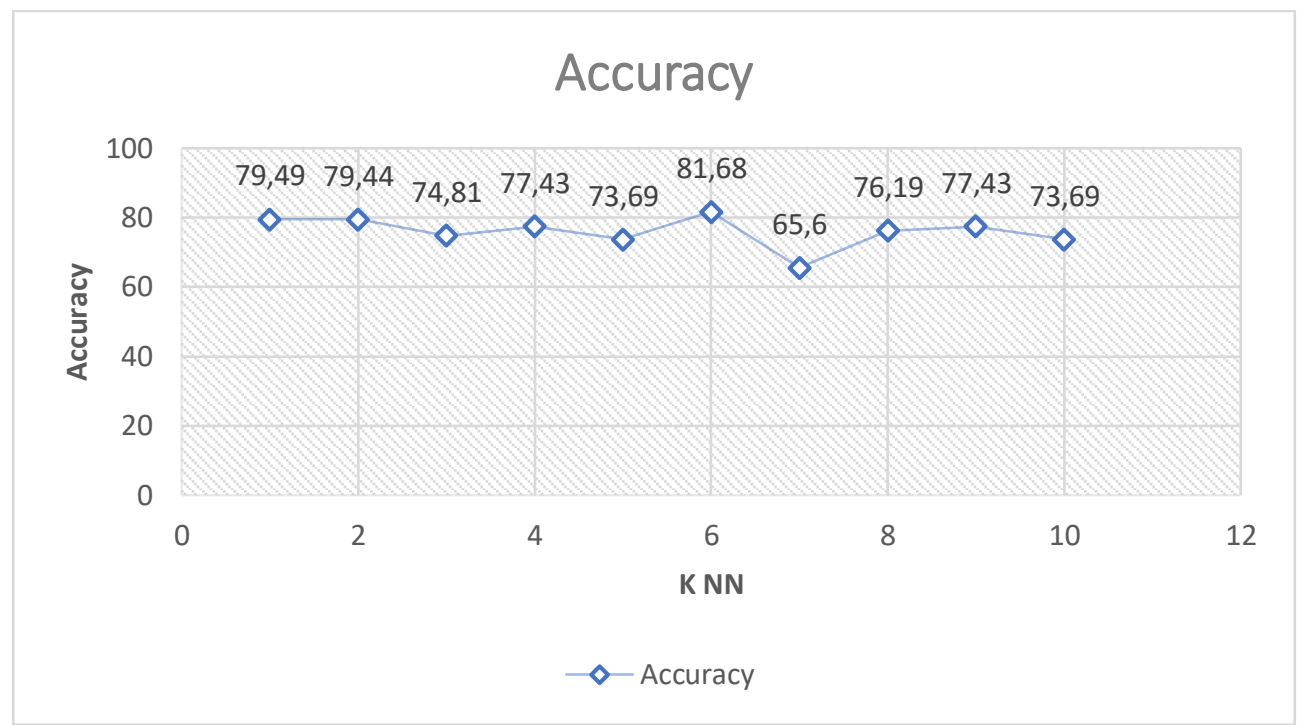

Figure 1: Instance based classifier Vs Accuracy

The above diagram shows that the highest accuracy value is $81.68 \%$ which is while applying the $=7 \mathrm{k}=6$ and the lowest accuracy value is $65.6 \%$ when apply the $\mathrm{k}=7$

Table 1: Instance based classifier Vs Precision

\begin{tabular}{|l|l|l|}
\hline S.No & K & Precision \\
\hline 1 & 1 & 81.68 \\
\hline 2 & 2 & 73.69 \\
\hline 3 & 3 & 78.14 \\
\hline 4 & 4 & 79.32 \\
\hline 5 & 5 & 79.49 \\
\hline 6 & 6 & 80.44 \\
\hline 7 & 7 & 74.81 \\
\hline 8 & 8 & 77.43 \\
\hline 9 & 9 & 73.69 \\
\hline 10 & 10 & 74.81 \\
\hline
\end{tabular}

The above table clearly shows that the instance based classifier is producing the precision value levels are different while doing the parameter tuning like $\mathrm{K}$ parameter. In this dataset we implemented the lazy classifier while $\mathrm{K}=1$ then the system gets a precision value is $81.68 \%$, while $\mathrm{K}=2$ then the system gets a precision value is $73.69 \%$, while $\mathrm{K}=3$ then the system gets a precision value is $78.14 \%$, while $K=4$ then the system gets a precision value is $79.32 \%$, while 
$\mathrm{K}=5$ then the system gets a precision value is $79.49 \%$, while $\mathrm{K}=6$ then the system gets a precision value is $80.44 \%$, while $\mathrm{K}=7$ then the system gets a precision value is $74.81 \%$, while $\mathrm{K}=8$ then the system gets a precision value is $77.43 \%$, while $\mathrm{K}=9$ then the system gets a precision value is $73.69 \%$, while $\mathrm{K}=10$ then the system gets a precision value is $74.81 \%$.

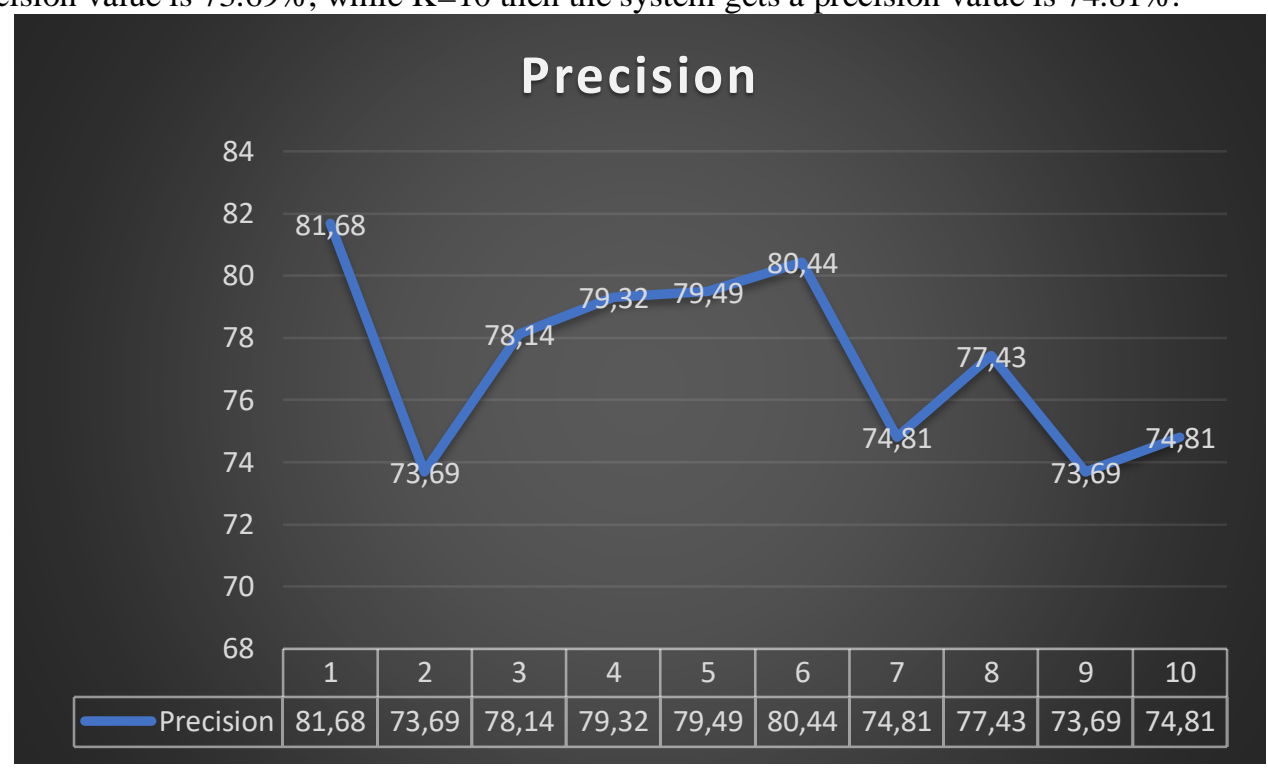

Figure 2: Instance based classifier Vs Precision

The above diagram clearly shows that the highest precision value is $81.68 \%$ while applying the $\mathrm{k}=1$ and very lowest precision value is $73.69 \%$ lies on $\mathrm{k}=2$ and $\mathrm{k}=9$.

Table 2: Instance based classifier Vs Recall

\begin{tabular}{|l|l|l|}
\hline S.No & K & Recall \\
\hline 1 & 1 & 79.43 \\
\hline 2 & 2 & 71.69 \\
\hline 3 & 3 & 80.68 \\
\hline 4 & 4 & 75.6 \\
\hline 5 & 5 & 75.19 \\
\hline 6 & 6 & 83.69 \\
\hline 7 & 7 & 74.81 \\
\hline 8 & 8 & 81.68 \\
\hline 9 & 9 & 65.76 \\
\hline 10 & 10 & 75.89 \\
\hline
\end{tabular}

The above table 2 clearly shows that the instance based classifier is producing the recall value levels are different while doing the parameter tuning like $\mathrm{K}$ parameter. In this dataset we implemented the lazy classifier while $\mathrm{K}=1$ then the system gets a recall value is $79.43 \%$, while $\mathrm{K}=2$ then the system gets a recall value is $71.69 \%$, while $\mathrm{K}=3$ then the system gets a recall value is $80.68 \%$, while $K=4$ then the system gets a recall value is $75.6 \%$, while $K=5$ then the system gets a recall value is $75.19 \%$, while $K=6$ then the system gets a recall value is $83.69 \%$, 
while $\mathrm{K}=7$ then the system gets a recall value is $74.81 \%$, while $\mathrm{K}=8$ then the system gets a recall value is $81.68 \%$, while $K=9$ then the system gets a recall value is $65.76 \%$, while $K=10$ then the system gets a recall value is $75.89 \%$.

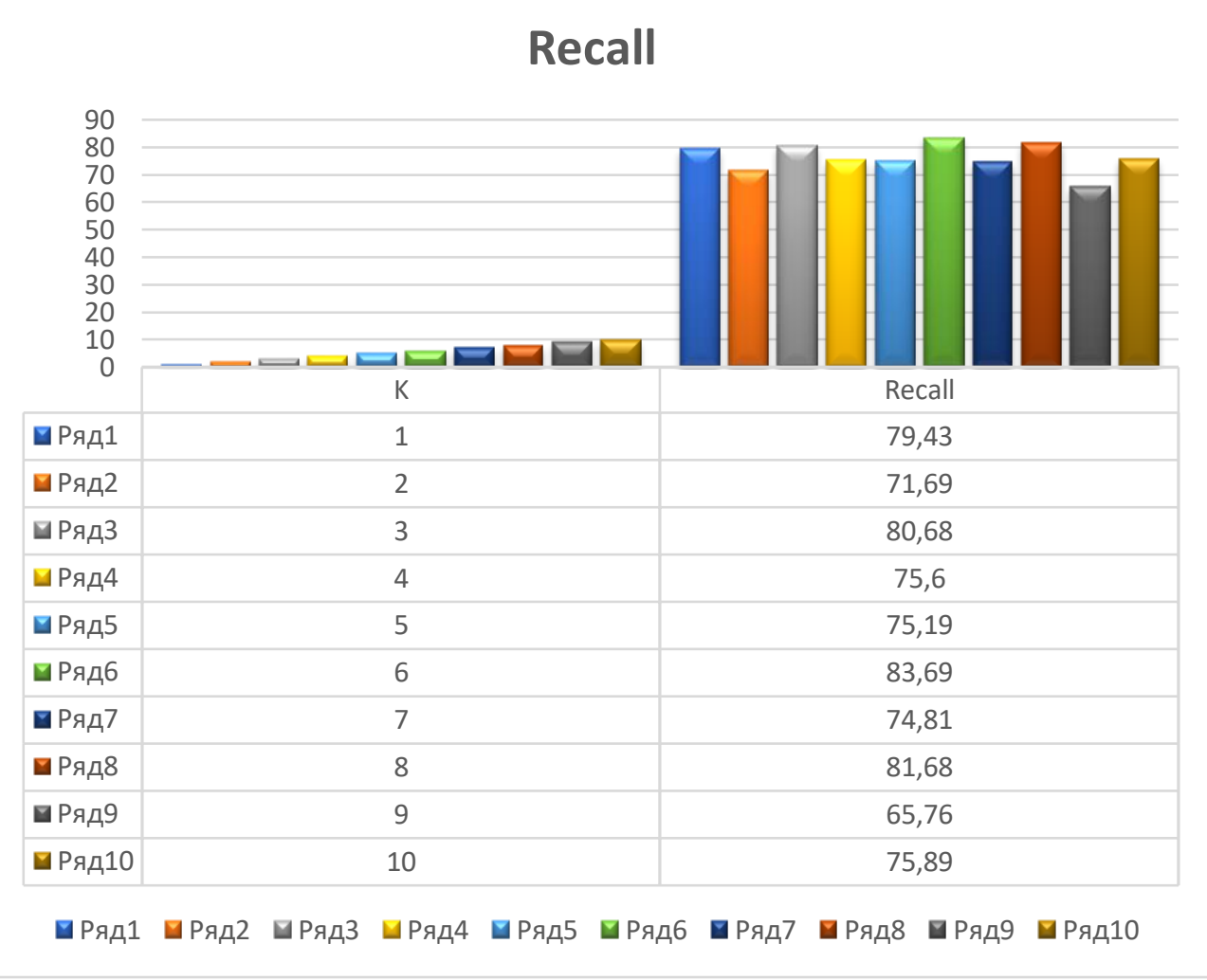

Figure 3: Instance based classifier Vs Recall

The above figure 3 the highest recall value is $83.69 \%$ which is produced by while applying the parameter $\mathrm{k}=6$, the lowest recall value is $65.76 \%$ while applying the parameter $\mathrm{k}=9$.

Table 3: Instance based classifier Vs Time

\begin{tabular}{|c|c|}
\hline $\mathbf{K}$ & Time taken to build model(In Seconds) \\
\hline 1 & 0.19 \\
\hline 2 & 0.21 \\
\hline 3 & 0.49 \\
\hline 4 & 0.25 \\
\hline 5 & 0.23 \\
\hline 6 & 0.91 \\
\hline 7 & 0.24 \\
\hline 8 & 0.14 \\
\hline 9 & 0.36 \\
\hline
\end{tabular}


The above table 3 clearly shows that the instance based classifier is taking the time consumption to build the model is different while doing the parameter tuning like $\mathrm{K}$ parameter. In this dataset we implement the lazy classifier while $K=1$ then the system takes a time consumption to build the model is 0.19 seconds, while $K=2$ then the system takes a time consumption to build the model is 0.21 seconds, while $\mathrm{K}=3$ then the system takes a time consumption to build the model is 0.49 seconds, while $\mathrm{K}=4$ then the system takes a time consumption to build the model is 0.25 seconds, while $K=5$ then the system takes a time consumption to build the model is 0.23 seconds, while $\mathrm{K}=6$ then the system takes an a time consumption to build the model is 0.91 seconds, while $\mathrm{K}=7$ then the system takes an a time consumption to build the model is 0.24 seconds, while $\mathrm{K}=8$ then the system takes an a time consumption to build the model is 0.14 seconds, while $\mathrm{K}=9$ then the system takes an a time consumption to build the model is 0.36 seconds, and finally while $\mathrm{K}=10$ then the system takes an a time consumption to build the model is 1.24 seconds.

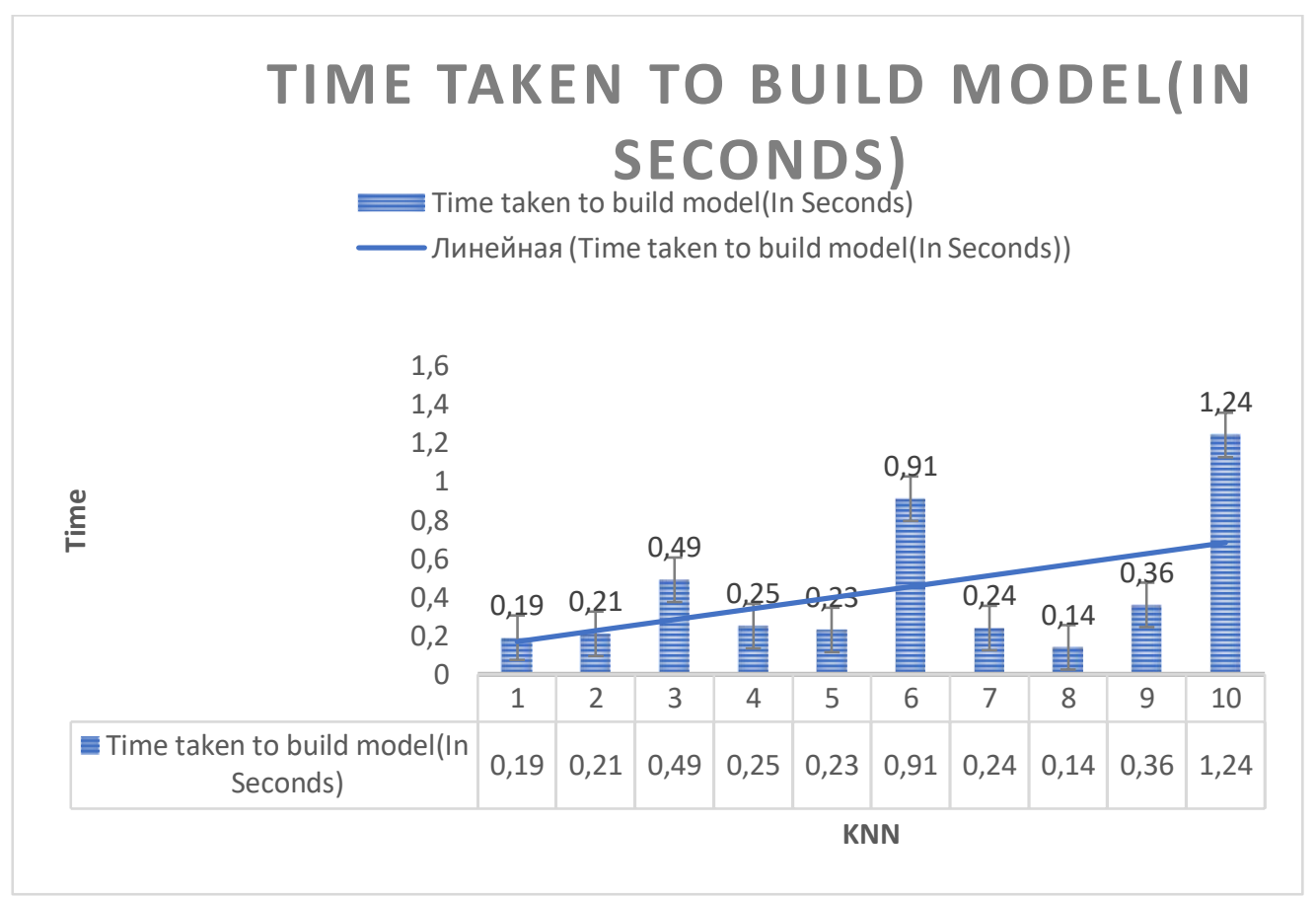

Figure 4: Instance based classifier Vs Time Consumption

The above figure 4 shows that the $\mathrm{K}=10$ model takes more time to build the model is while applying the $\mathrm{k}=10$ and very low time consumption model is $\mathrm{k}=8$. 


\section{Conclusions}

The System concludes that the highest accuracy value is $81.68 \%$ which is while applying the $=7 \mathrm{k}=6$ and the lowest accuracy value is $65.6 \%$ when apply the $\mathrm{k}=7$. The highest precision value is $81.68 \%$ while applying the $\mathrm{k}=1$ and very lowest precision value is $73.69 \%$ lies on $\mathrm{k}=2$ and $\mathrm{k}=9$. The highest recall value is $83.69 \%$ which is produced by while applying the parameter $\mathrm{k}=6$, the lowest recall value is $65.76 \%$ while applying the parameter $\mathrm{k}=9$. The $\mathrm{K}=10$ model takes more time to build the model is while applying the $\mathrm{k}=10$ and very low time consumption model is $\mathrm{k}=8$.

\section{References}

[1] Robert H. Cofield Total shoulder arthroplasty with the neer prosthesis J Bone Joint Surgery, 66 (6) (1984), pp. 899-906

[2] Jia Deng, Wei Dong, Richard Socher, Li-Jia Li, Kai Li, Li Fei-FeiImagenet: a large-scale hierarchical image database 2009 IEEE Conference on Computer Vision and Pattern Recognition, Ieee (2009), pp. 248-255

[3] Simonyan Karen, Zisserman Andrew, Very deep convolutional networks for large-scale image recognition. ArXiv Preprint ArXiv:1409.1556; 2014.

[4] Kaiming He, Xiangyu Zhang, Shaoqing Ren, Jian Sun Deep residual learning for image recognition Proceedings of the IEEE Conference on Computer Vision and Pattern Recognition (2016), pp. 770-778

[5] Gao Huang, Zhuang Liu, Laurens Van Der Maaten, Kilian Q. Weinberger Densely connected convolutional networks Proceedings of the IEEE Conference on computer vision and pattern recognition (2017), pp. 4700-4708

[6] Barret Zoph, Vijay Vasudevan, Jonathon Shlens, Quoc V. Le Learning transferable architectures for scalable image recognition Proceedings of the IEEE Conference on computer vision and pattern recognition (2018), pp. 8697-8710

[7] EuijoonAhn, Ashnil Kumar, Jinman Kim, Changyang Li, Dagan Feng, Michael Fulham X-ray image classification using domain transferred convolutional neural networks and local sparse spatial pyramid 2016 IEEE 13th International Symposium on Biomedical Imaging (ISBI), IEEE (2016), pp. 855-858

[8] Juan Wang, Huanjun Ding, FateMehAzamian, Brian Zhou, Carlos Iribarren, SabeeMolloi, Pierre Baldi Detecting cardiovascular disease from mammograms with deep learning IEEE Trans Med Imaging, 36 (5) (2017), pp. 1172-1181

[9] Shuchao Pang, Zhezhou Yu, Mehmet A Zhezhou, Orgun A novel end-to-end classifier using domain transferred deep convolutional neural networks for biomedical images Computer Methods Programs Biomed, 140 (2017), pp. 283-293

[10] Gregor Urban, PriyamTripathi, TalalAlkayali, Mohit Mittal, FaridJalali, William Karnes, Pierre Baldi Deep learning localizes and identifies polyps in real time with $96 \%$ accuracy in screening colonoscopy Gastroenterology, 155 (4) (2018), pp. 1069-1078

[11] Juan Wang, Zhiyuan Fang, Ning Lang, Huishu Yuan, Su. Min-Ying, Pierre Baldi A multiresolution approach for spinal metastasis detection using deep siamese neural networks Computers Biol Med, 84 (2017), pp. 137-146

[12] P. Baldi Deep learning in biomedical data science Ann Rev Biomed Data Sci, 1 (2018), pp. 181 205

[13] Gregor Urban, Kevin Bache, Duc TT Phan, Agua Sobrino, Alexander K. Shmakov, Stephanie J. Hachey, et al. Deep learning for drug discovery and cancer research: automated analysis of vascularization images IEEE/ACM Trans ComputBiolBioinform, 16 (3) (2018), pp. 1029-1035 
[14] Aaron Defazio, Francis Bach, Simon Lacoste-Julien Saga: A fast incremental gradient method with support for non-strongly convex composite objectives Advances in Neural Information Processing Systems (2014), pp. 1646-1654

[15] Leo Breiman Random forests Mach Learn, 45 (1) (2001), pp. 5-32

[16] Jerome H. Friedman Greedy function approximation: a gradient boosting machine Ann Statistics (2001), pp. 1189-1232

[17] Thomas M. Cover, Peter E. Hart, et al. Nearest neighbor pattern classification IEEE Trans Inform Theory, 13 (1) (1967), pp. 21-27

[18] http://sfsu-dspace.calstate.edu/handle/10211.3/204021

[19] https://www.sciencedirect.com/science/article/pii/S2001037019304179?via\%3Dihub

[20] T. Vijayan , M. Sangeetha, A. Kumaravel \& B. Karthik (2020): FeatureSelection for Simple Color Histogram Filter based on Retinal Fundus Images for DiabeticRetinopathy Recognition, IETE Journal of Research, DOI: 10.1080/03772063.2020.1844082.

[21] D. S. Vijayan, A. Leema Rose, S. Arvindan, J. Revathy, C. Amuthadevi, "Automation systems in smart buildings: a review", Journal of Ambient Intelligence and Humanized Computing https://doi.org/10.1007/s12652-020-02666-9

[22] Vijayan T, Sangeetha M, A. Kumaravel, Karthik B, "Gabor filter and machine learning based diabetic retinopathy analysis and detection", Microprocessors and Microsystems,2020. https://doi.org/10.1016/j.micpro.2020.103353.

[23] Vijayan T, SangeethaM, Karthik B, "Trainable WEKA Segmentation of Retinal Fundus Images for Global Eye Disease Diagnosis Application," International Journal of Emerging Trends in

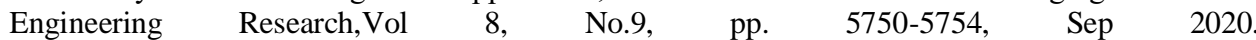
https://doi.org/10.30534/ijeter/2020/136892020

[24] C. Amuthadevi, D. S. Vijayan, Varatharajan Ramachandran, "Development of air quality monitoring (AQM) models using different machine learning approaches", Journal of Ambient Intelligence and Humanized Computing, https://doi.org/10.1007/s12652-020-02724-2

[25] Vijayan T, Sangeetha M, A. Kumaravel, Karthik B, "Fine Tuned VGG19 Convolutional Neural Network Architecture for Diabetic Retinopathy Diagnosis," Indian Journal of Computer Science and Engineering (IJCSE), Vol. 11, No. 5, pp. 615-622 Sep-Oct 2020. DOI: 10.21817/indjcse/2020/v11i5/201105266.

[26] Vijayan T, Sangeetha M, Karthik B, "Efficient Analysis of Diabetic Retinopathy on Retinal Fundus Images using Deep Learning Techniques with Inception V3 Architecture,” Journal of Green Engineering, Vol 10, Issue 10, pp. 9615-9625. Oct 2020 